\title{
Concussions and Brain Injuries in Children: United States, 2020
}

\author{
Lindsey I. Black, M.P.H., and Benjamin Zablotsky, Ph.D.
}

\section{Key findings}

\section{Data from the National Health Interview Survey}

- In $2020,6.8 \%$ of children aged 17 years and under had ever had symptoms of a concussion or brain injury.

- Non-Hispanic White children were more likely than children of other race and Hispanic-origin groups to have ever had symptoms of a concussion or brain injury.

- The percentage of children aged 17 years and under who had ever had a diagnosis of a concussion or brain injury by a health care provider was $3.9 \%$.

- Compared with their peers, boys $(4.7 \%)$ and non-Hispanic White children $(5.2 \%)$ were more likely to have ever had a diagnosis of a concussion or brain injury.
A concussion is a mild type of traumatic brain injury (1). Previous studies using national data have shown that concussion prevalence can differ by survey methodology and question wording (2). The 2020 National Health Interview Survey (NHIS) included questions on concussion to measure both symptoms and diagnosis from a health care provider to provide a more complete understanding of the public health burden, because children with mild injuries may not see a doctor or receive a diagnosis. This report presents national estimates of lifetime symptomatology and health care professional diagnoses of concussions or brain injuries as reported by a knowledgeable adult, usually a parent, in children aged $0-17$ years using data from the 2020 NHIS.

\section{The percentage of children who had ever had symptoms of a concussion or brain injury varied by age group.}

Figure 1. Percentage of children aged 0-17 years who ever had symptoms of concussion or brain injury, by age group: United States, 2020

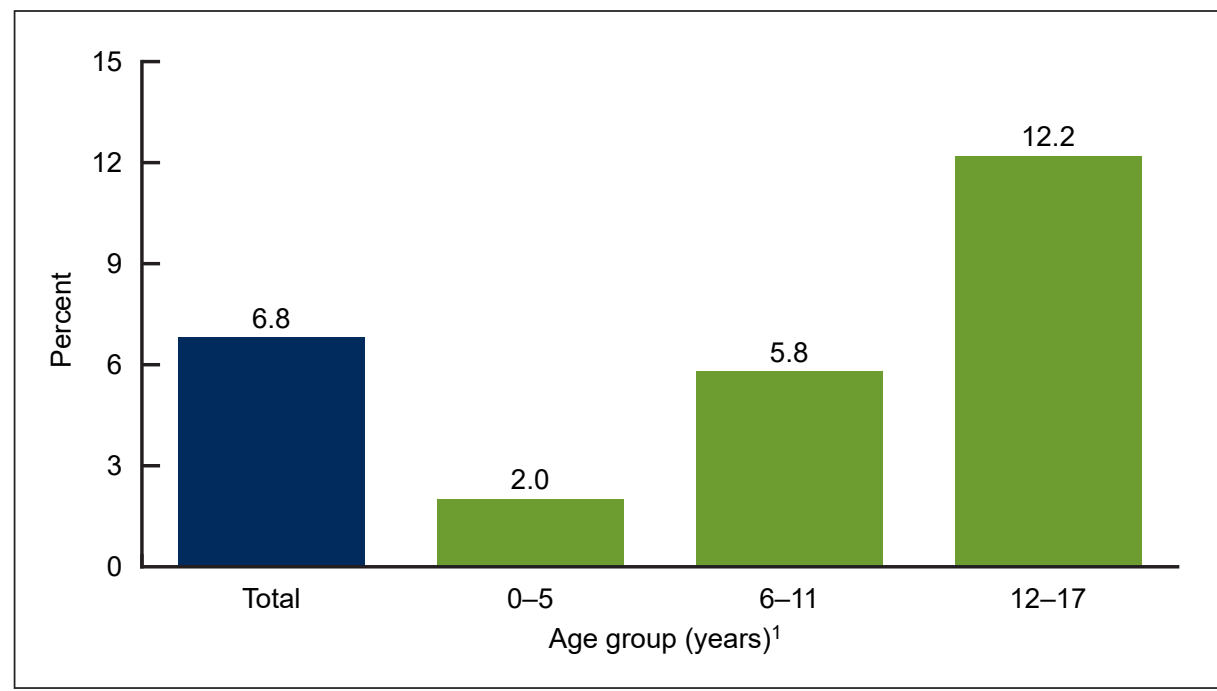

${ }^{1}$ Significant increasing linear trend with age $(p<0.05)$.

NOTES: "Symptoms of concussion or brain injury" is a summary based on positive responses to any of the symptoms questions (ever knocked out or lost consciousness; dazed or had a gap in memory; or headaches, vomiting, blurred vision, or changes in mood or behavior). Children could have more than one type of symptom; see Definitions for more information. Estimates are based on household interviews of a sample of the U.S. civilian noninstitutionalized population. Access data table for Figure 1 at: https://www.cdc.gov/nchs/data/databriefs/db423-tables.pdf\#1.

SOURCE: National Center for Health Statistics, National Health Interview Survey, 2020.

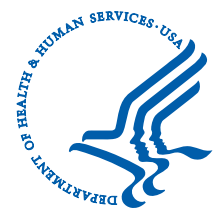




\section{NCHS Data Brief a No. 423 a December 2021}

In 2020, $6.8 \%$ of children had ever had symptoms of a concussion or brain injury in their lifetime (Figure 1). The percentage of children who had ever had symptoms of a concussion or brain injury increased with age, from $2.0 \%$ in children aged 5 years and under to $12.2 \%$ in children aged 12-17.

\section{The percentage of children who had ever had symptoms of a concussion or brain injury varied by race and Hispanic origin.}

Non-Hispanic White children $(8.8 \%)$ were more likely than children from other race and Hispanic-origin groups to have ever had symptoms of a concussion or brain injury (Figure 2). Moreover, Hispanic children (5.6\%) were more likely than non-Hispanic Black (2.6\%) and non-Hispanic Asian (3.0\%) children to have ever had symptoms of a concussion or brain injury. Additionally, boys (7.7\%) were more likely than girls (5.9\%) to have ever had symptoms of a concussion or brain injury, although this difference was not significant.

Figure 2. Percentage of children aged 0-17 years who ever had symptoms of concussion or brain injury, by sex and race and Hispanic origin: United States, 2020

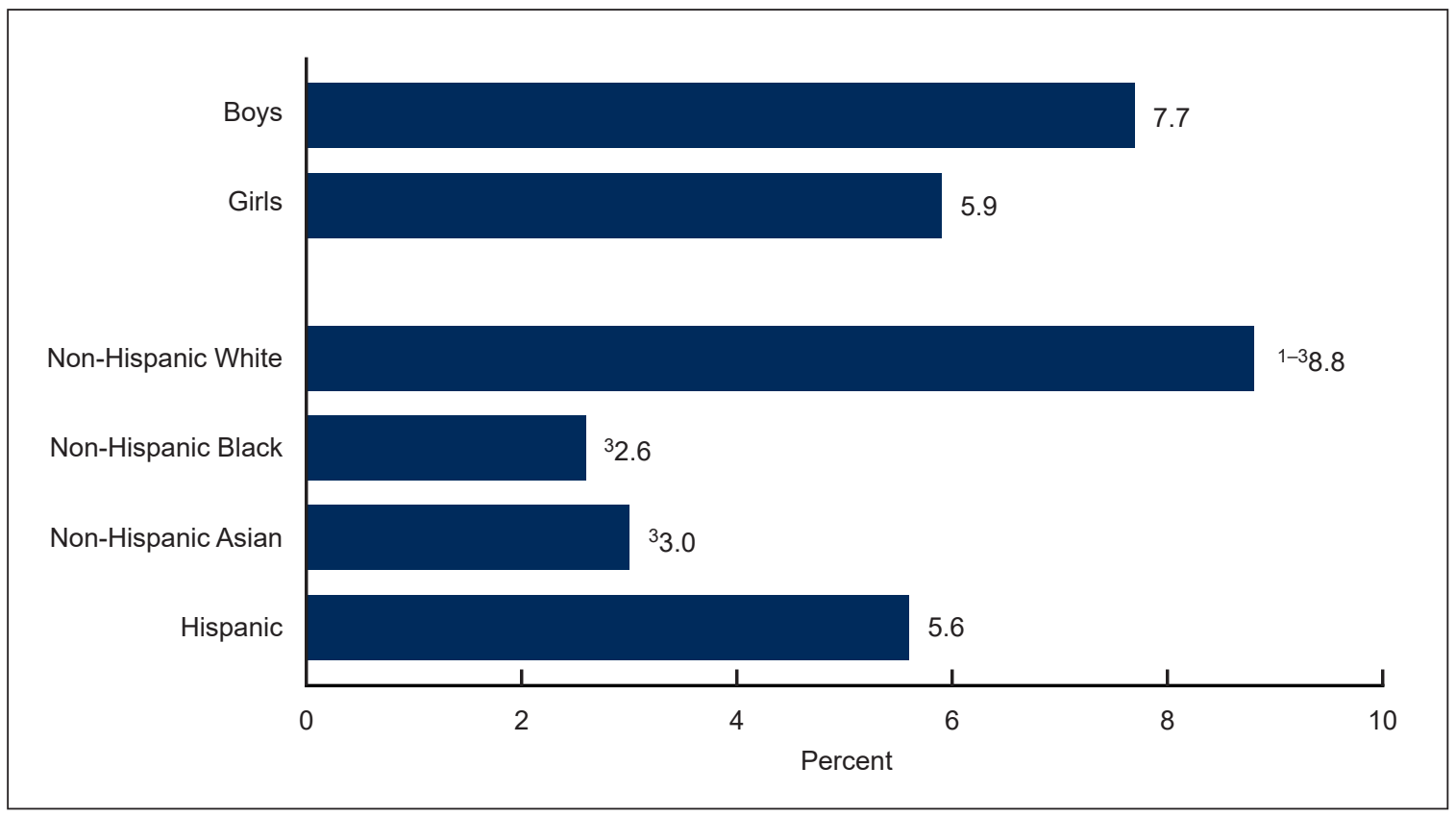

${ }^{1}$ Signficantly different from non-Hispanic Black children $(p<0.05)$

${ }^{2}$ Signficantly different from non-Hispanic Asian children $(p<0.05)$

${ }^{3}$ Signficantly different from Hispanic children $(p<0.05)$

NOTES: "Symptoms of concussion or brain injury" is a summary based on positive responses to any of the symptoms questions (ever knocked out or lost consciousness; dazed or had a gap in memory; or headaches, vomiting, blurred vision, or changes in mood or behavior). Children could have more than one type of symptom; see Definitions for more information. Estimates are based on household interviews of a sample of the U.S. civilian noninstitutionalized population. Access data table for Figure 2 at: https://www.cdc.gov/nchs/data/databriefs/db423-tables.pdf\#2.

SOURCE: National Center for Health Statistics, National Health Interview Survey, 2020. 


\section{NCHS Data Brief a No. 423 a December 2021}

\section{The percentage of children who had received a diagnosis of a concussion or brain injury by a health care professional in their lifetime varied by age.}

Overall, $3.9 \%$ of children had received a diagnosis of a concussion or brain injury by a health care professional in their lifetime (Figure 3). The percentage of children who had received a diagnosis of a concussion or brain injury increased with age and was approximately nine times higher in children aged $12-17$ years (8.3\%) compared with children aged 5 and under $(0.9 \%)$.

Figure 3. Percentage of children aged 0-17 years who received a diagnosis of concussion or brain injury by a health care professional, by age group: United States, 2020

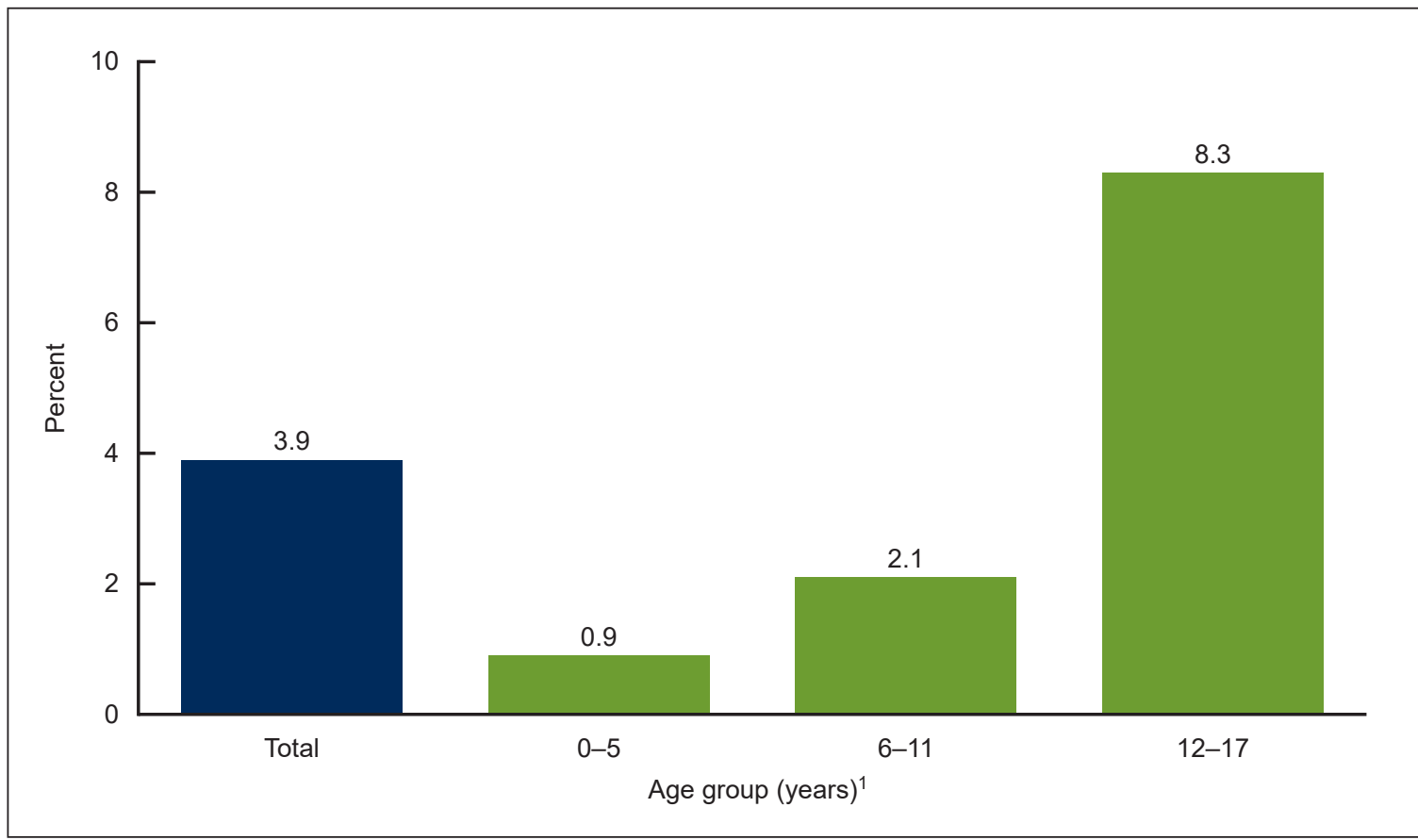

${ }^{1}$ Significant increasing linear trend with age $(p<0.05)$

NOTES: "Received a diagnosis of concussion or brain injury" is based on respondents who indicated that the Sample Child had ever been checked for a concussion or brain injury by a health care professional and received a diagnosis of concussion or brain injury; all children are included in the denominator. Estimates are based on household interviews of a sample of the U.S. civilian noninstitutionalized population. Access data table for Figure 3 at: https://www.cdc.gov/nchs/data/databriefs/db423-tables.pdf\#3.

SOURCE: National Center for Health Statistics, National Health Interview Survey, 2020. 


\section{NCHS Data Brief a No. 423 a December 2021}

\section{The percentage of children who had received a diagnosis of a concussion or brain injury by a health care professional in their lifetime varied by sex and race and Hispanic origin.}

Boys (4.7\%) were more likely than girls (3.1\%) to have received a diagnosis of a concussion or brain injury by a health care professional in their lifetime (Figure 4). Non-Hispanic White children (5.2\%) were more likely than children from all other race and Hispanic-origin groups to have received a diagnosis of a concussion or brain injury by a health care professional in their lifetime. Similarly, Hispanic children (3.1\%) were more likely than non-Hispanic Asian $(1.1 \%)$ and non-Hispanic Black (1.5\%) children to have ever received such a diagnosis, although the difference between Hispanic and non-Hispanic Black children was not significant.

Figure 4. Percentage of children aged 0-17 years who received a diagnosis of concussion or brain injury by a health care professional, by sex and race and Hispanic origin: United States, 2020

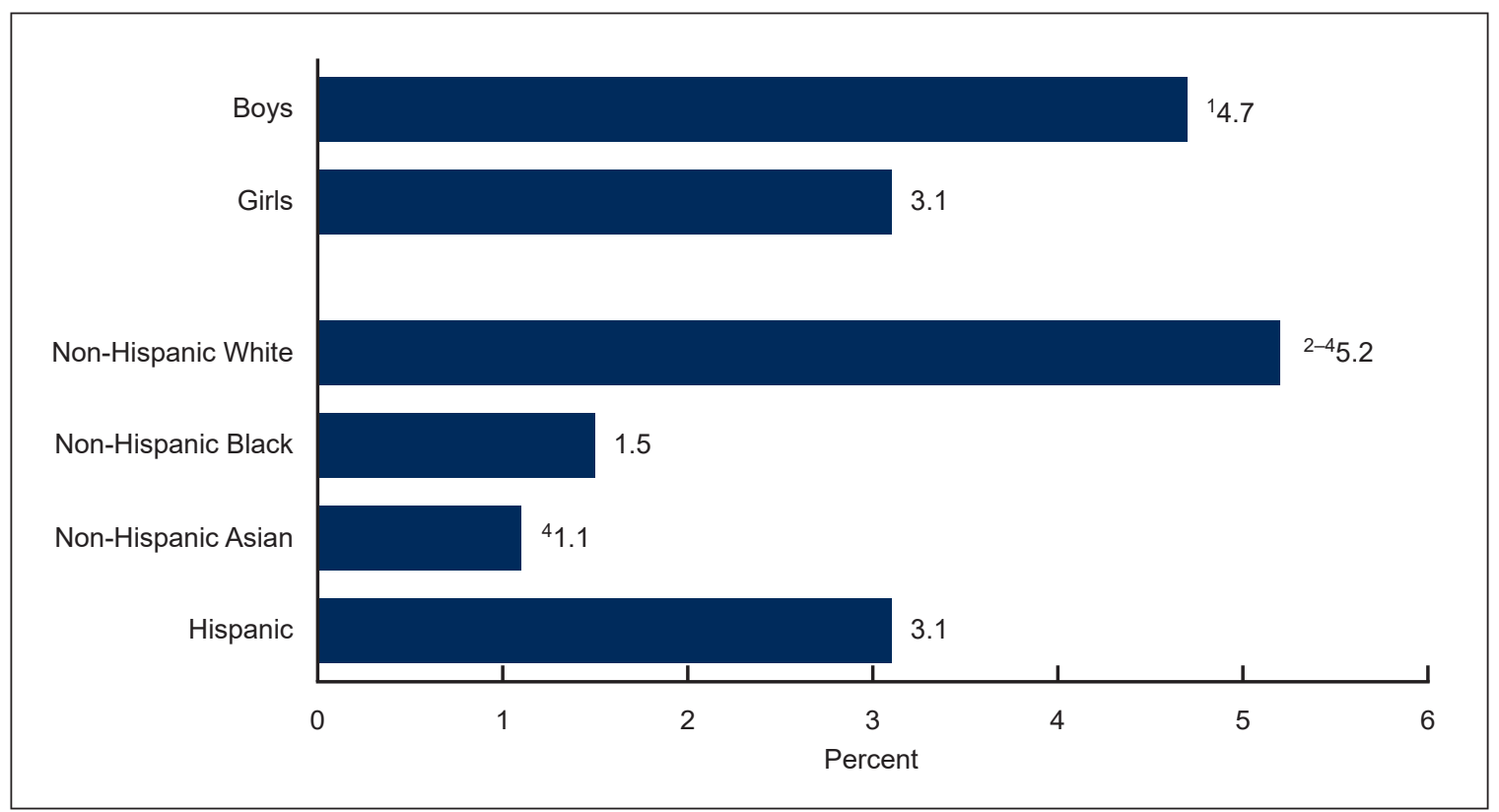

${ }^{1}$ Significantly different from girls $(p<0.05)$.

${ }^{2}$ Significantly different from non-Hispanic Black children $(p<0.05)$.

${ }^{3}$ Significantly different from non-Hispanic Asian children $(p<0.05)$.

${ }^{4}$ Significantly different from Hispanic children $(p<0.05)$.

NOTES: "Received a diagnosis of a concussion or brain injury" is based on respondents who indicated that the Sample Child had ever been checked for

concussion or brain injury by a health care professional and received a diagnosis of concussion or brain injury; all children are included in the denominator.

Estimates are based on household interviews of a sample of the U.S. civilian noninstitutionalized population. Access data table for Figure 4 at:

https://www.cdc.gov/nchs/data/databriefs/db423-tables.pdf\#4.

SOURCE: National Center for Health Statistics, National Health Interview Survey, 2020. 


\section{Summary}

This report describes national estimates for symptoms and diagnosis of a concussion or brain injury for children aged $0-17$ years by age group, sex, and race and Hispanic origin using data from NHIS. Overall, $6.8 \%$ of children had ever had symptoms of a concussion or brain injury and $3.9 \%$ were ever diagnosed with a concussion or brain injury. For both symptoms and diagnosis, prevalence increased with increasing age. The prevalence of symptoms was higher for boys than girls, and non-Hispanic White children were more likely to have ever had symptoms of a concussion or brain injury than all other race and Hispanic-origin groups examined. Similarly, the prevalence of diagnosed concussion or brain injury was higher for boys than girls, and non-Hispanic White children were more likely than all other race and Hispanic-origin groups to have ever been diagnosed with a concussion or brain injury.

\section{Definitions}

Ever had symptoms of a concussion or brain injury: A composite measure based on an affirmative response to any of the following survey questions: 1) "As a result of a blow or jolt to the head, has [Sample Child] ever been knocked out or lost consciousness?"; 2) "As a result of a blow or jolt to the head, has [Sample Child] ever been dazed or had a gap in his/her memory?"; or 3) "As a result of a blow or jolt to the head, has [Sample Child] had headaches, vomiting, blurred vision, or changes in mood or behavior?" Respondents who indicated "yes" to the first symptom question did not receive the second and third symptom questions.

Ever received a diagnosis of a concussion or brain injury: Based on an affirmative response to the survey question, "Did a doctor, nurse, athletic trainer, or other health professional ever say that [Sample Child] had a concussion or brain injury?" Respondents who answered "no" to the preceding survey question, "Has [Sample Child] ever been checked for a concussion or brain injury by a doctor, nurse, athletic trainer, or other health professional?" were not asked about diagnosis and were included in the denominator as "no" for this question.

Race and Hispanic origin: Children categorized as Hispanic may be of any race or combination of races. Children categorized as non-Hispanic White, non-Hispanic Black, and non-Hispanic Asian indicated one race only. Estimates for non-Hispanic children of other races are not shown. 


\section{NCHS Data Brief a No. 423 a December 2021}

\section{Data source and methods}

NHIS is a nationally representative household survey of the U.S. civilian noninstitutionalized population. It is conducted continuously throughout the year by the National Center for Health

Statistics (NCHS). Interviews are typically conducted in respondents' homes, but follow-ups to complete interviews may be conducted over the telephone. Due to the COVID-19 pandemic, data collection procedures in 2020 were disrupted: From April to June, interviews were conducted by telephone only, and from July to December, interviews were attempted by telephone first with follow-ups to complete interviews by personal visit. For more information about NHIS, visit https://www.cdc.gov/nchs/nhis.htm.

Point estimates and the corresponding confidence intervals for this analysis were calculated using SAS-callable SUDAAN software (3) to account for the complex sample design of NHIS. All estimates are based on parent or guardian report and meet NCHS data presentation standards for proportions (4). Differences between percentages were evaluated using two-sided significance tests at the 0.05 level. Linear trends by age group were evaluated using Proc Descript, poly option.

\section{About the authors}

Lindsey I. Black and Benjamin Zablotsky are with NCHS’ Division of Health Interview Statistics. 


\section{NCHS Data Brief a No. 423 a December 2021}

\section{References}

1. National Center for Injury Prevention and Control. What is a concussion? Available from: https://www.cdc.gov/headsup/basics/concussion_whatis.html.

2. Haarbauer-Krupa J, Lebrun-Harris LA, Black LI, Veliz P, Daugherty J, Desrocher R, et al. Comparing prevalence estimates of concussion/head injury in U.S. children and adolescents in national surveys. Ann Epidemiol 54:11-20. 2021.

3. RTI International. SUDAAN (Release 11.0.3) [computer software]. 2018.

4. Parker JD, Talih M, Malec DJ, Beresovsky V, Carroll M, Gonzalez JF Jr, et al. National Center for Health Statistics data presentation standards for proportions. National Center for Health Statistics. Vital Health Stat 2(175). 2017. 
Centers for Disease Control and Prevention

\section{NCHS Data Brief $\square$ No. 423 a December 2021}

Keywords: traumatic brain injury • pediatric $\bullet$ National Health Interview Survey (NHIS)

\section{Suggested citation}

Black LI, Zablotsky B. Concussions and brain injuries in children: United States, 2020. NCHS Data Brief, no 423. Hyattsville, MD: National Center for Health Statistics. 2021. DOI: https://dx.doi.org/10.15620/ cdc: 111174 .

\section{Copyright information}

All material appearing in this report is in the public domain and may be reproduced or copied without permission; citation as to source, however, is appreciated.

\section{National Center for Health Statistics}

Brian C. Moyer, Ph.D., Director Amy M. Branum, Ph.D., Associate Director for Science

Division of Health Interview Statistics Stephen J. Blumberg, Ph.D., Director Anjel Vahratian, Ph.D., M.P.H., Associate Director for Science

For e-mail updates on NCHS publication releases, subscribe online at: https://www.cdc.gov/nchs/email-updates.htm.

For questions or general information about NCHS:

Tel: 1-800-CDC-INFO (1-800-232-4636)

TTY: $1-888-232-6348$

Internet: https://www.cdc.gov/nchs

Online request form: https://www.cdc.gov/info

ISSN 1941-4927 Print ed.

ISSN 1941-4935 Online ed. 